\title{
Spectral measure function separability and reflectionless potentials
}

\author{
Eric A. Kincanon * \\ Gonzaga University, Spokane, WA USA \\ *Corresponding author E-mail: kincanon@gonzaga.edu
}

\begin{abstract}
Both reflectionless potentials and special conditions on the spectral measure function have been well studied in inverse scattering theory. This short paper considers a spectral measure function that is separable and shows that it is equivalent to the potential being reflectionless.
\end{abstract}

Keywords: Inverse Scattering; Reflection less Potentials.

\section{Introduction}

A potential V(r) can be determined from the reflection coefficient $\mathrm{R}(\mathrm{k})$ of a scattered wave using the well-known method of inverse scattering. For problems in one dimension, this is done by using the Gelfand-Levitan equation [1-3]:

$$
K(r, s)+G(r, s)+\int_{-\infty}^{r} K(r, t) G(t, s) d t=0
$$

Where $\mathrm{G}(\mathrm{r}, \mathrm{s})$, the spectral measure function, is the Fourier transform of the reflection coefficient $\mathrm{R}(\mathrm{k})$ :

$$
G(r, s)=\frac{1}{2 \pi} \int_{-\infty}^{+\infty} R(k) e^{-i k(r+s)} d k+\text { Bound State Terms }
$$

And $\mathrm{K}(\mathrm{r}, \mathrm{s})$ is related to the potential by:

$V(r)=-2 \frac{d K(r, r)}{d r}$

The typical procedure is to find $\mathrm{V}(\mathrm{r})$ by taking the Fourier transform of $\mathrm{R}(\mathrm{k})$ to get $\mathrm{G}(\mathrm{r}, \mathrm{s})$ and then solve (1) for $\mathrm{K}(\mathrm{r}, \mathrm{s})$. Then (3) can be used to find the potential. The challenge arises in solving (1). This is typically done by successive iteration [4].

Reflection less potentials are of ongoing interest [5] and their connection to separable spectral measure functions have also been studied. [6-8] This paper considers the separability of G(r,s) and shows that it must have a particular mathematical form that matches that of reflectionless potentials. It is therefore shown that separability of $\mathrm{G}(\mathrm{r}, \mathrm{s})$ and the reflectionless condition are equivalent.

\section{Calculations}

Requiring that $\mathrm{G}(\mathrm{r}, \mathrm{s})$ is separable means that:

$G(r+s)=g(r) g(s)$

Through a series of simple mathematical steps it can be shown that this condition is only satisfied by an exponential function. First the partial of $G(r, s)$ with respect to $r$ is taken: $\frac{\partial \mathrm{G}(\mathrm{r}+\mathrm{s})}{\partial \mathrm{r}}=\frac{\partial \mathrm{g}(\mathrm{r})}{\partial \mathrm{r}} \mathrm{g}(\mathrm{s})$

Considering the definition of $\mathrm{G}(\mathrm{r}, \mathrm{s})$, given by equation (2), one can see that $\mathrm{G}(\mathrm{r}, \mathrm{s})=\mathrm{G}(\mathrm{r}+\mathrm{s})$. Letting $\mathrm{z}=\mathrm{r}+\mathrm{s}$, it is clear that:

$\frac{\partial \mathrm{G}(\mathrm{z})}{\partial \mathrm{r}}=\frac{\partial \mathrm{G}(\mathrm{r}, \mathrm{s})}{\partial \mathrm{z}} \frac{\partial \mathrm{z}}{\partial \mathrm{r}}$

Therefore, combining (5) and (6) gives:

$\frac{\partial \mathrm{G}(\mathrm{z})}{\partial \mathrm{z}}=\frac{\partial \mathrm{g}(\mathrm{r})}{\partial \mathrm{r}} \mathrm{g}(\mathrm{s})$

Alternatively, the above procedure could be carried out and instead take the partial with respect to s. Since $G(r, s)$ is symmetric with respect to $r$ and $s$ this gives:

$$
\frac{\partial \mathrm{G}(\mathrm{z})}{\partial \mathrm{z}}=\frac{\partial \mathrm{g}(\mathrm{s})}{\partial \mathrm{s}} \mathrm{g}(\mathrm{r})
$$

Therefore, seeing that right hand sides of (7) and (8) must be equal gives that:

$\frac{\partial \mathrm{g}(\mathrm{s})}{\partial \mathrm{s}} \mathrm{g}(\mathrm{r})=\frac{\partial \mathrm{g}(\mathrm{r})}{\partial \mathrm{r}} \mathrm{g}(\mathrm{s})$

Or,

$\frac{1}{g(s)} \frac{\partial g(s)}{\partial s}=\frac{1}{g(r)} \frac{\partial g(r)}{\partial r}$

The right hand side of (10) is only dependent on $r$ and the left hand side is only dependent on s, so each must equal a constant, therefore

$\frac{1}{\mathrm{~g}(\mathrm{~s})} \frac{\partial \mathrm{g}(\mathrm{s})}{\partial \mathrm{s}}=\mathrm{c}$

This has the well-known solution:

$\mathrm{g}(\mathrm{x})=A \mathrm{e}^{\mathrm{cx}}$

Applying (12) to (4) yields: 
$\mathrm{G}(\mathrm{r}+\mathrm{s})=A \mathrm{e}^{\mathrm{c}(\mathrm{r}+\mathrm{s})}$

Now consider the relationship between $G(r, s)$ and $R(k)$. As noted in the introduction:

$\mathrm{G}(\mathrm{r}, \mathrm{s})=\frac{1}{2 \pi} \int_{-\infty}^{+\infty} \mathrm{R}(\mathrm{k}) \mathrm{e}^{-\mathrm{ik}(\mathrm{r}+\mathrm{s})} \mathrm{dk}+$ Bound State Terms

Bound state terms have the general form:

$\sum_{i} C_{i} \emptyset_{i}(r) \emptyset_{i}(s)$

A thorough discussion of the challenges of using bound state terms of the Gelfand-Levitan equation can be found in [3].

Considering (14) one sees that if $\mathrm{R}(\mathrm{k})$ is zero, $\mathrm{G}(\mathrm{r}, \mathrm{s})$ consists of only bound state terms. And since (15) is separable, it is not surprising that reflectionless potentials have a separable $G(r, s)$. However, one might wonder if this is an if and only if condition. Though the consideration of (14) and (15) gives the conclusion that $G(r, s)$ is separable if $R(k)$ is zero, it is not clear that $G(r, s)$ is not separable for other cases. This would be the case if the Fourier Transform part of (14) is separable. The calculation that yields (13), though, has demonstrated that the only separable $G(r, s)$ must be an exponential function.

Therefore, a G(r,s) that is separable must have the form of equation (12). Combining this with (14), one can see that $\mathrm{R}(\mathrm{k})$ must be zero. To do this the bound state terms can be ignored, since the only interest is with the separable Fourier transform. Taking the inverse transform of (14) gives:

$$
R(k)=\frac{1}{2 \pi} \int_{-\infty}^{+\infty} G(z) e^{i k(z)} d z
$$

Where $z=r+s$, as previously defined. Considering (16) with

$G(z)=A e^{c(z)}$

One sees that the Fourier Transform diverges, which is an unacceptable physical solution.

Therefore, G(r,s) cannot be separable for cases when $R(k)$ is not zero and the equivalence of separability and reflectionless potentials has been shown.

\section{Conclusion}

This paper has demonstrated two things. First, that the only possible separable form of the spectral measure function, $G(r, s)$ is an exponential. Second, that the only way for $G(r, s)$ to have this form is if the reflection coefficient, $\mathrm{R}(\mathrm{k})$, is zero. Therefore, the separability of $\mathrm{G}(\mathrm{r}, \mathrm{s})$ is equivalent to having a reflectionless potential.

\section{References}

[1] I.M. Gelfand, B.M. Levitan, on the determination of a differential equation by its spectral function, Dokl. Akad. Nauk. USSR 77 (1951) 557-560.

[2] I.M. Gelfand, B.M. Levitan, on the determination of a differential equation by its spectral measure function, Izv. Akad. Nauk. SSR 15 (1951) 309-360.

[3] K. Chadan, P.C. Sabatier, Inverse Problems in Quantum Scattering Theory, Springer-Verlag, NewYork, 1977. https://doi.org/10.1007/978-3-662-12125-2.

[4] R. Jost, W. Kohn, on the relation between phase shift energy levels and the potential, Danske Vid. Selsk. Math. Fys. 27 (1953) 3-19.

[5] J. Lekner, Reflectionless eigenstates of the $\operatorname{sech}^{2}$ potential, Am. J. Phys. 75 (2007) 1151-1157. https://doi.org/10.1119/1.2787015.

[6] E. Kincanon, An Orthogonal Set Composed from the Functions $\mathrm{e}^{\mathrm{nx}}$, Applied Mathematics and Computation, 41, (1991) 69-75. https://doi.org/10.1016/0096-3003(91)90107-X

[7] E. Kincanon, Approximate solution to the Gelfand-Levitan equation, Applied Mathematics and Computation, 53 (1993) 121-128. https://doi.org/10.1016/0096-3003(93)90097-X.
[8] E. Kincanon, Spectral measure function separability and reflectionless potentials, Applied Mathematics and Computation, 123 (2001) 409-412. https://doi.org/10.1016/S0096-3003(00)00086-2. 